\title{
A novel class of copper(II)- and zinc(II)-bound non-steroidal anti-inflammatory drugs that inhibits acute inflammation in vivo
}

\author{
Rajesh Puranik ${ }^{1,6}$, Shisan Bao ${ }^{1,5^{*}}$, Antonio M. Bonin², Ravinder Kaur², Jane E. Weder², Llewellyn Casbolt ${ }^{3}$,
} Trevor W. Hambley ${ }^{2}$, Peter A. Lay ${ }^{2}$, Philip J. Barter ${ }^{1}$ and Kerry-Anne Rye ${ }^{1,4}$

\begin{abstract}
Background: The ability of Zn(II) and Cu(II) metal complexes of non-steroidal anti-inflammatory drugs (NSAIDs) to inhibit acute arterial inflammation in vivo has been studied.

Results: When acute vascular inflammation was induced in normocholesterolemic New Zealand White rabbits by inserting a non-occlusive silastic collar around the common carotid artery, a single oral dose of $\mathrm{Cu}(\mathrm{II})$-indomethacin (Cu(II)Indo, $3 \mathrm{mg} / \mathrm{kg}$ ) administered by laparotomy achieved a $67 \%(8.2 \pm 1.7 \mathrm{vs} .2 .7 \pm 0.4$ image units, $p<0.05)$ reduction in endothelial expression of vascular cell adhesion molecule-1 (VCAM-1) but did not inhibit endothelial intercellular adhesion molecule (ICAM-1) expression significantly. Treatment with $\mathrm{Cu}(\mathrm{II})$-acemetacin (Cu(II)ACM, $3 \mathrm{mg} / \mathrm{kg}$ ) led to a profound $88 \%(8.2 \pm 1.7$ vs. $1.0 \pm 0.5$ image units, $p<0.01)$ reduction in endothelial VCAM-1 expression but did not inhibit ICAM-1 expression, while treatment with Zn(II)-acemetacin (Zn(II)ACM, 3 mg/kg) led to an $84 \%(19.3 \pm 1.0$ vs. $3.1 \pm 1.2$ image units, $p<0.01$ ) reduction in endothelial ICAM-1 expression and did not inhibit VCAM-1 expression. No adverse gastric, hepatic or renal effects were observed in treated animals.

Conclusion: These findings provide the "proof of concept" that this novel class of drug, where there is complexation of NSAIDs with metal ions, has substantial anti-inflammatory effects in an animal model of acute vascular inflammation with the possibility of low rates of adverse effects.
\end{abstract}

Keywords: NSAIDs, Indomethacin, Copper(II) indomethacin, Copper(II) ACM, Zinc(II) ACM

\section{Background}

Non-steroidal anti-inflammatory drugs (NSAIDs) are amongst the most commonly prescribed drugs in the community, specifically for the treatment of a wide variety of inflammatory conditions [1]. However, patients receiving non-selective NSAIDs often experience abdominal discomfort, while some develop serious gastro-intestinal (GI) complications such as ulceration, bleeding, perforation or obstruction [2].

\footnotetext{
*Correspondence: bob.bao@sydney.edu.au

${ }^{5}$ Discipline of Pathology, D17, The School of Medical Sciences and Bosch

Institute, The University of Sydney, Sydney, NSW 2006, Australia

Full list of author information is available at the end of the article
}

It was, therefore postulated that with the advent of selective cyclooxygenase-2 (COX-2) inhibitors, the number of NSAID-related gastric complications would be reduced [3]. However, current evidence demonstrates that selective COX2 inhibitors ('coxibs') have important cardiovascular side-effects that include increased risk for myocardial infarction, stroke, heart failure and hypertension [4]. Due to concerns regarding CV risks, the American Heart Association recommends that treatment with coxibs should be limited to patients in whom no appropriate alternatives can be found, and then only in the lowest dose for the shortest duration necessary [5]. A subsequent joint study with the American College of Cardiology Foundation and the American College of Gastroenterology 
recommended concomitant therapy with proton pump inhibitors such as esomeprazole, to offset the GI damage from NSAID use [6]. According to the Pharmaceutical Benefits Scheme, esomeprazole use represents the fourth highest cost to Australia in terms of national health expenditure.

An alternative approach, used in veterinary practice [7], is the complexation of NSAIDs with metal ions, which results in enhanced gastric protection [7] compared with the parent NSAID. Another potential strategy for developing NSAIDs with attenuated GI side effects is to use acemetacin $(\mathrm{ACMH})$, a glycolic ester of indomethacin (IndoH) with inherent antiinflammatory activity and better gastric tolerability compared with IndoH [8]. In addition, ACMH has a reportedly safer pharmacokinetic profile in the elderly [8]. Therefore, it is an ideal candidate for complexation with biologically relevant metals such as copper $(\mathrm{Cu})$ and zinc $(\mathrm{Zn})$ [9].

Given the pressing need for alternative safer NSAIDtype drugs that could be applied to a large range of medical indications, we have investigated the impact of IndoH and copper bound Indo ( $\mathrm{Cu}$ (II)Indo) with that of copper bound $(\mathrm{Cu}(\mathrm{II}) \mathrm{ACM})$, and zinc bound ACM ( $\mathrm{Zn}(\mathrm{II}) \mathrm{ACM})$ on acute arterial inflammation in vivo in the New Zealand White (NZW) rabbit.

\section{Results and discussion}

The mean plasma concentrations of total cholesterol, HDL cholesterol and apoA-I in the rabbits were $0.84 \pm 0.1,0.66 \pm 0.05 \mathrm{mM} / \mathrm{l}$, and $0.76 \pm 0.07 \mathrm{mg} /$ $\mathrm{ml}$, respectively. At the time of sacrifice, there was no measurable effect on the concentration of any of these parameters with any of the treatments that were administered.

\section{Effect of treatment on endothelial VCAM-1 expression (Fig. 1a-g)}

There was marked up regulation in endothelial expression of VCAM-1 24 h post-carotid collar insertion (Fig. 1a, b, $p<0.01)$. Treatment with IndoH $(3 \mathrm{mg} / \mathrm{kg}$ ) led to a $75 \%$ decrease in endothelial VCAM-1 expression $(8.2 \pm 1.7$ vs. $2.1 \pm 1.4$ image units) $(p<0.05)$ (Fig. 1c) Treatment with $\mathrm{Cu}(\mathrm{II})$ Indo $(3 \mathrm{mg} / \mathrm{kg}$ ) led to a $67 \%$ $(8.2 \pm 1.7$ vs. $2.7 \pm 0.4$ image units $)(p<0.05)$ reduction in endothelial VCAM-1 expression (Fig. 1d). Treatment with $\mathrm{Cu}(\mathrm{II}) \mathrm{ACM}(3 \mathrm{mg} / \mathrm{kg})$ resulted in an $88 \%(8.2 \pm 1.7$ vs. $1.0 \pm 0.5$ image units $)(p<0.01)$ reduction of endothelial VCAM-1 expression (Fig. 1e). The $39 \%$ (8.2 \pm 1.7 vs. $5.0 \pm 1.4$ image units, $p=0.19$ ) reduction in VCAM-1 expression, which resulted from treatment with $\mathrm{Zn}$ (II) ACM (3 mg/kg), did not achieve statistical significance (Fig. 1f).

\section{Effect of treatment on endothelial ICAM-1 expression} (Fig. 2a-g)

Insertion of a carotid collar markedly up-regulated endothelial ICAM-1 expression (Fig. 2a, b, $p<0.01$ ). Treatment with IndoH $(3 \mathrm{mg} / \mathrm{kg})$ led to a $79 \%$ decrease in endothelial ICAM-1 expression $[19.3 \pm 1.0$ vs. $4.1 \pm 1.4$ image units $(p<0.01)$ Fig. 2c]. Treatment with $\mathrm{Cu}(\mathrm{II})$ Indo $(3 \mathrm{mg} / \mathrm{kg})$ led to a $28 \%(19.3 \pm 1.0$ vs. $13.9 \pm 6.3$ image units, $p=0.43$ ) decrease in ICAM-1 expression (Fig. 2d). There was a $43 \%(19.3 \pm 1.0$ vs. $11.0 \pm 3.6$ image units, $p=0.07)$ decrease in ICAM-1 expression with $\mathrm{Cu}(\mathrm{II})$ ACM (3 mg/kg), which was not statistically significant (Fig. 2e), whereas treatment with $\mathrm{Zn}$ (II)ACM (3 mg/ $\mathrm{kg})$ led to a $84 \%(19.3 \pm 1.0$ vs. $3.1 \pm 1.2$ image units $)$ $(p<0.01)$ decrease in ICAM-1 expression (Fig. 2f).

\section{Toxicity data}

The active ingredients were well tolerated by all animals. Both macroscopic and microscopic immunohistochemical analyses revealed no evidence of gastric, hepatic or renal toxicity. Examination of the entire length of the stomach, small and large bowel showed no evidence of ulceration, gastrointestinal bleeding or inflammation. On histology there was no evidence of acute hepatitis or nephritis in any of the animals.

In this work, we have demonstrated for the first time that $\mathrm{Cu}$ and $\mathrm{Zn}$ bound anti-inflammatory agents can also be effective treatments for acute vascular inflammation in vivo. The degree of anti-inflammatory activity observed was found to be similar to that achieved with Indo $\mathrm{H}$ at equivalent to the FDA recommended maximum therapeutic dose (RMTD). Furthermore, this new class of anti-inflammatory agents showed no signs of organ (stomach, small and large intestine, liver, and kidney) acute toxicity at this dose.

Specifically, we observed that $\mathrm{Cu}(\mathrm{II})$-bound Indo and ACM achieved a comparable level of inhibition of endothelial VCAM-1 expression as observed after treatment with IndoH. Interestingly, $\mathrm{Zn}$ (II)-bound ACM was able to decrease expression of endothelial ICAM-1 substantially, despite a lack of significant inhibition of VCAM-1 expression, which indicated that $\mathrm{Zn}(\mathrm{II})$ ACM inhibits inflammation independent of the canonical nuclear factor- $\mathrm{kB}(\mathrm{NF}-\mathrm{\kappa} B)$ pathway. Importantly, the results of this study are comparable to a similar study in which the anti-inflammatory effects of the atheroprotective agent lipid-free apolipoprotein A-I (apoA-I) were investigated [10]. However the degree of inhibition of VCAM and ICAM achieved warrants larger studies including mechanistic studies at the MRNA level to further delineate these effects in vivo.

Treatments with both NSAIDs and coxibs are associated with significant rates of GI and cardiovascular 

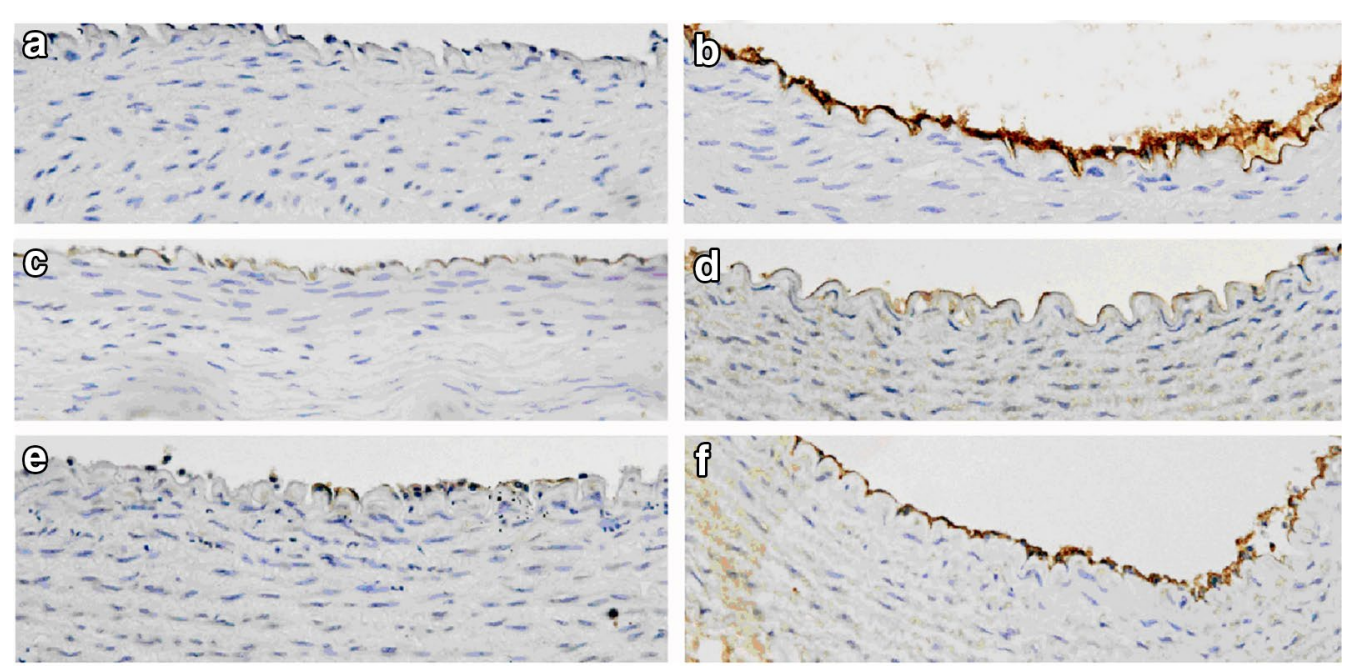

VCAM-1 Expression

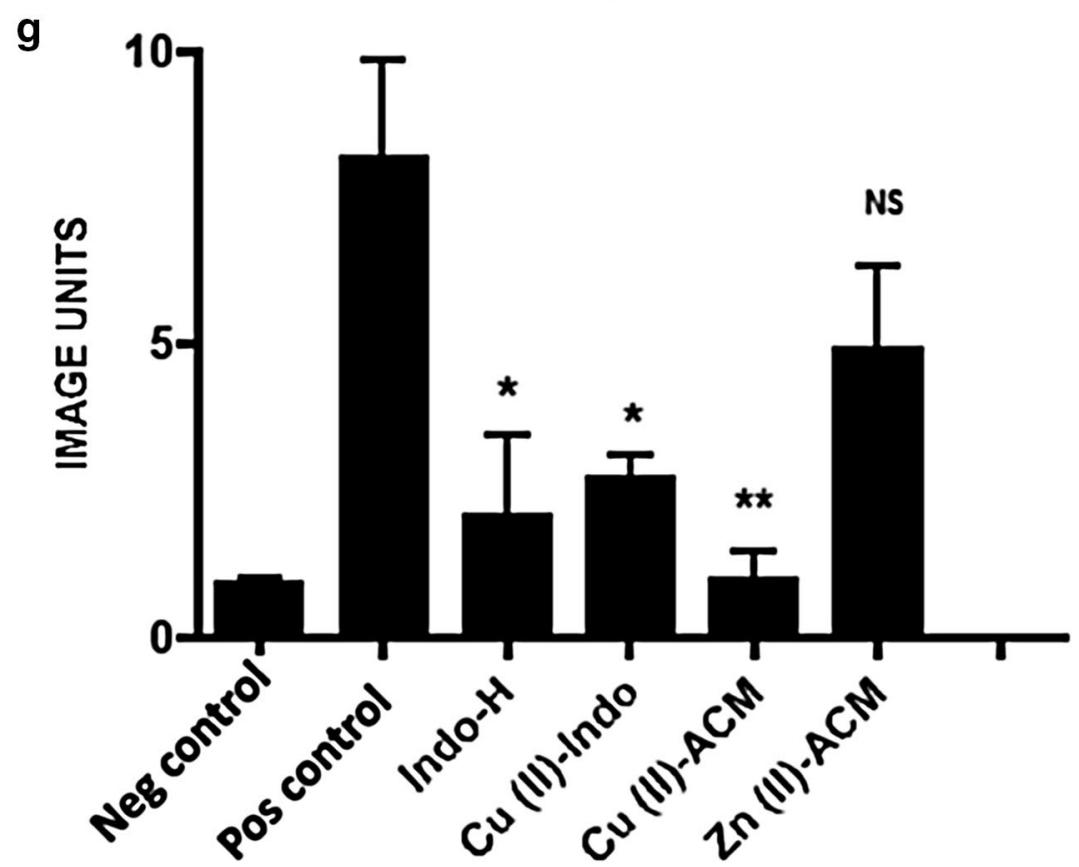

Fig. 1 Effect of treatment on endothelial expression of VCAM-1. Normocholesterolemic NZW rabbits were treated with either saline, IndoH (3 mg/ $\mathrm{kg}), \mathrm{Cu}(\mathrm{II}) \mathrm{Indo}(3 \mathrm{mg} / \mathrm{kg}), \mathrm{Cu}(I)$ ACM $(3 \mathrm{mg} / \mathrm{kg})$ or Zn(II)ACM (3 mg/kg) ( $n=4$ per group) at the time of peri-arterial collar insertion. The animals were sacrificed $24 \mathrm{~h}$ after collar insertion. Representative sections of carotid arteries stained for VCAM- 1 expression ( $\times 40$ magnification) are shown. a a representative control, non-collared artery from a saline-treated animal, $\mathbf{b}$ a collared artery section from a saline-treated animal, $\mathbf{c}$ a section of a collared artery where the animal is treated with IndoH, $\mathbf{d}$ a section of a collared artery from an animal treated with $\mathrm{Cu}(\mathrm{II}) \mathrm{Indo}$, e a collared artery section from an animal treated with $\mathrm{Cu}(\mathrm{II}) \mathrm{ACM}$ and $\mathbf{f}$ a section of a collared artery from an animal is treated with $\mathrm{Zn}(\mathrm{II}) \mathrm{ACM}$. In $\mathbf{g}$ the results are expressed graphically as mean $\pm \operatorname{SEM}\left({ }^{*} p<0.05 ;{ }^{* *} p<0.01\right)$

(CV) pathology [2-4]. It was initially postulated that selective coxibs may specifically decrease GI adverse side effects. However, it is now apparent that, despite a reduction in GI complications with selective coxibs, serious CV events may occur [11]. Therefore, there is pressing clinical need for the development of effective anti-inflammatory agents with a wider safety and therapeutic window. 


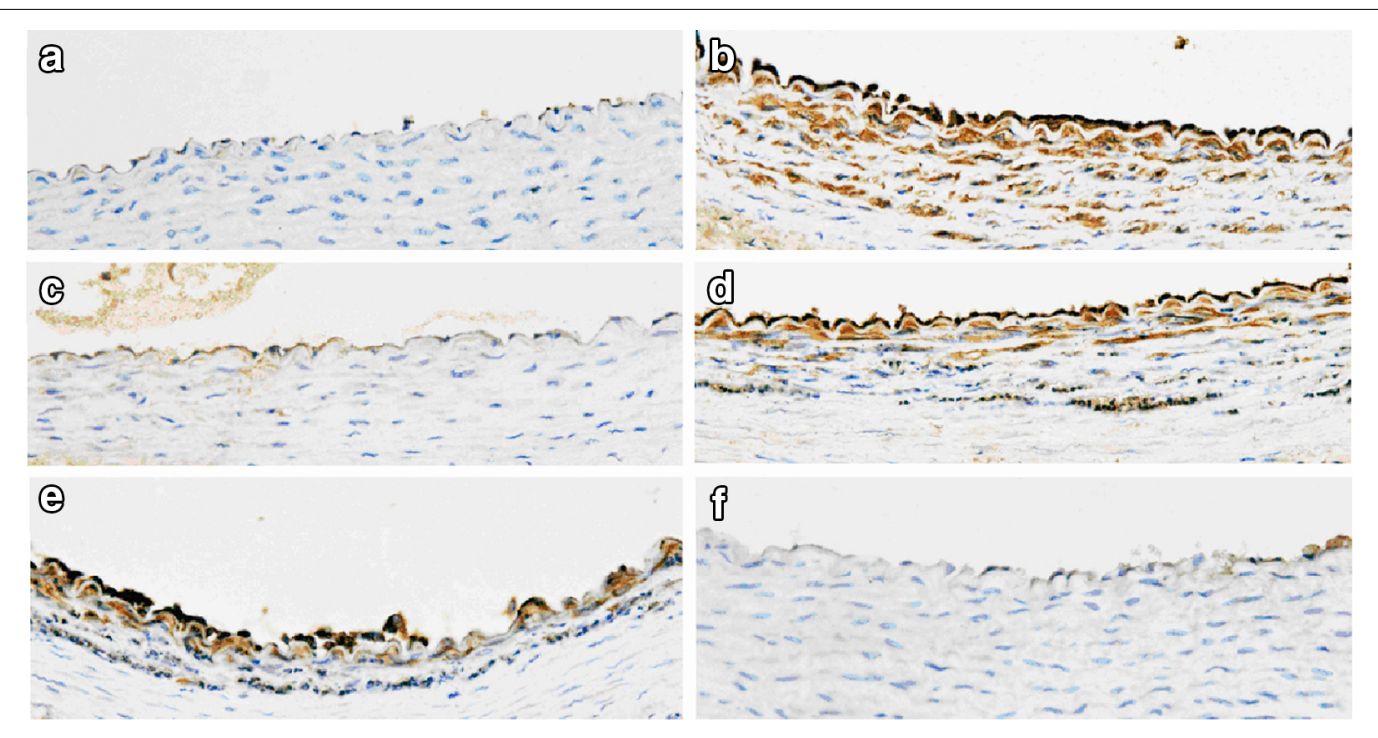

ICAM-1 Expression

g

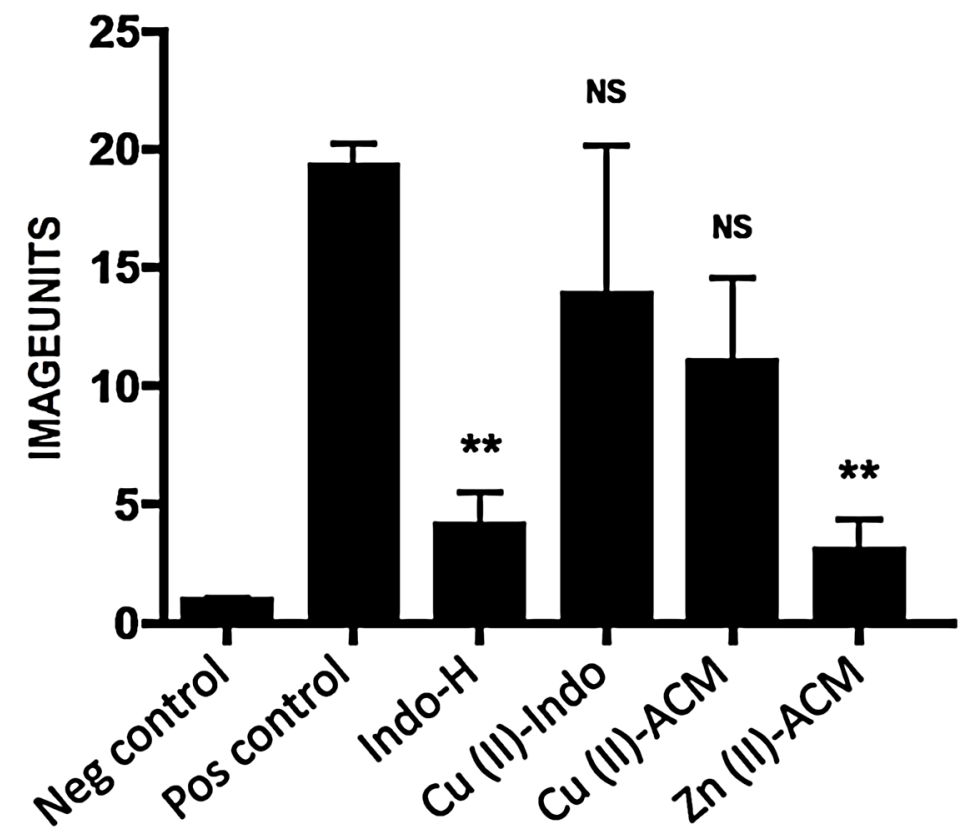

Fig. 2 Effect of treatment on endothelial expression of ICAM-1. A non-occlusive collar was inserted around the left common carotid artery of normolipidemic NZW rabbits, as described in the legend to Fig. 1. Representative sections of carotid arteries stained for ICAM-1 expression ( $\times 40$ magnification) are shown in the panels. $\mathbf{a}$ a representative section from a control, non-collared artery from a saline-treated animal, $\mathbf{b}$ a collared artery section from a saline-treated animal, $\mathbf{c}$ a section of a collared artery from an animal treated with indomethacin (IndoH), $\mathbf{d}$ a section of a collared artery from a Cu(II)-Indo-treated animal, e a section of a collared artery from an animal treated with $\mathrm{Cu}(\mathrm{II})-\mathrm{ACM}$ and $\mathbf{f}$ a section of a collared artery from an animal treated with $\mathrm{Zn}(\mathrm{II})-\mathrm{ACM}$. In $\mathbf{g}$ the results are expressed graphically as mean $\pm \mathrm{SEM}\left({ }^{* *} p<0.01\right)$

An early strategy to circumvent the inherent toxicity of NSAID use was by means of NSAID complexation with metals, particularly $\mathrm{Cu}$. This arose from the research of Hangarter in the mid-twentieth century [12], and the hypothesis of Sorenson in the 1990s that the in vivo activity of NSAIDs might be due to binding with endogenous $\mathrm{Cu}$ [13], and the gastric-sparing effect of the $\mathrm{Cu}$ (II)NSAID complexes. Many other $\mathrm{Cu}(\mathrm{II})$-NSAID complexes 
have since been synthesised [7, 13] as well as those of other metals, such as $\mathrm{Zn}$ (II) [14], for the assessments of their anti-inflammatory, anti-cancer and anti-diabetic effects.

Acemetacin (systematic name: 2-[2-(1-(4-chlorobenzoyl)5-methoxy-2-methyl- $1 H$-indol-3-yl)acetoxy] acetic acid) is an IndoH prodrug with inherent anti-inflammatory activity. ACMH has been on the market for over 35 years and although its GI-protective advantage relative to IndoH is well-established [8], relatively little is known about its mode of action [15]. ACMH is known to be rapidly absorbed and converted to IndoH in vivo, with complete biotransformation within $1 \mathrm{~h}$ of ingestion. It has been suggested that the reduced GI toxicity of ACMH results from its lack of stimulation of leucocyte-endothelial adherence within mesenteric venules, a crucial event in the genesis of NSAID gastropathy [15], hence it is considered a suitable candidate for evaluation of its anti-inflammatory activity and non-ulcerogenic potential in a variety of conditions and diseases. Further, expansion of the current protocol to include ACMH and/or other market available NSAIDs would also be of value.

Preliminary investigations of the $\mathrm{Cu}$ (II) complex of ACM provide useful pointers to the relative safety and efficacy and dose-response of the complex compared with the parent NSAID. A comparable level of inhibition of cell adhesion molecule expression by IndoH and the metal complexes of Indo and ACM is indicative of the anti-inflammatory potency of all of these classes of drugs. Both VCAM-1 and ICAM-1 are important early markers of atherogenesis, and serum levels of soluble VCAM-1 and soluble ICAM-1 independently predict CV events [16]. It is likely that the anti-inflammatory effects of these compounds are, at least in part, explained by inhibition of NF- $\mathrm{kB}$, where variable magnitudes of inhibition of VCAM-1 and ICAM-1 can be attributed to the differing effects on the regulators of their expression, especially in relation to the ACM compounds.

For clinical applications, it is necessary to consider the safety of profiles of these drugs, which are associated with their mode(s) of action. $\mathrm{Cu}$ (II)Indo exhibits an enzymatic anti-oxidant-like superoxide dismutase (SOD) activity, with an $I C_{50}$ value $(0.04 \mu \mathrm{M})$ [17], which is comparable to other potent SOD-mimetic Cu complexes [18]. Indo complexes with either $\mathrm{Cu}(\mathrm{II})$ or $\mathrm{Zn}$ (II) showed an improvement in GI safety compared to the parent drug in rats and horses [17], but this was more evident for the $\mathrm{Cu}$ (II) complex and its SOD-like activity may contribute to the reduced GI toxicity. The clinically protective effects of the $\mathrm{Cu}$ complexes are further supported by the lack of GI, hepatic and renal toxicities observed in the NZW rabbit model used in the current study. Moreover, in Phase 1 human clinical trials for the treatment of proctitis, no toxicity was observed with $\mathrm{Cu}(\mathrm{II})$ Indo where normally the GI toxicity of NSAIDs would preclude treatment for this condition [19].

A limitation of this study was the small numbers of animals per treatment group. This maybe of relevance to the differential inhibitory effects previously discussed. However, the degree of inhibition of VCAM-1 and ICAM-1 achieved warrants larger studies to further delineate these effects in vivo; in particular investigation of effects on vascular inflammation of a combined therapy using both $\mathrm{Cu}$ (II)-NSAID and Zn(II)-NSAID compared with the parent NSAID alone. Also as the model used in our experiments was one of acute vascular inflammation, further experiments investigating chronic vascular insults leading to atherosclerotic changes in the vessel wall are required. Finally, in regards to toxicity data, it is unlikely that major adverse effects would be observed after a single dose of an NSAID in a small number of animals, as used in our study. In future studies we plan to expand the dose range and duration, as well as measure liver concentrations of copper and plasma concentrations of ceruloplasmin.

In conclusion, we have demonstrated for the first time that a single oral dose of $\mathrm{Cu}$ (II) and $\mathrm{Zn}$ (II)-bound Indolike compounds have marked anti-inflammatory effects in an acute model of vascular inflammation in vivo. This study establishes the "proof-of-concept" that such a class of drug can be effective with the additional benefit of no apparent GI, hepatic and renal toxicity. The ability of these compounds to inhibit the expression of adhesion molecules provides the initial evidence to further develop these drugs for human conditions of acute and chronic vascular inflammation and investigate their application in the treatment of other inflammation based disease states.

\section{Methods}

\section{Synthesis and formulation of test compounds}

Acemetacin, IndoH and zinc acetate dihydrate were obtained from Sigma Chemical Co. (St. Louis, MO, USA). Copper(II) acetate monohydrate (99.0\%) was obtained from Ajax Chemicals. All the chemicals were used without further purification for synthesis of the following active ingredients for testing: $\mathrm{Cu}$ (II)Indo, $\mathrm{Cu}$ (II) ACM, (Fig. 3a) and Zn(II) ACM [9] (Fig. 3b). Tetraglycol and sorbitan monooleate (Span $80^{\circledR}$ ) were obtained from Sigma Chemical Co. (St. Louis, MO, USA). Fumed silica $\left(\right.$ Aerosil ${ }^{\circledR}$ ) and medium chain triglyceride (MCT) oil were supplied by NatureVet Pty. Ltd. The MCT organogel was prepared by dissolving the active ingredient in tetraglycol (40\% w/w), Span $80(20 \% \mathrm{w} / \mathrm{w})$ and MCT oil (adjusted to $100 \% \mathrm{w} / \mathrm{w})$, and heating the mixture to $55-60{ }^{\circ} \mathrm{C}$. Aerosil was added to the still warm solution, and mixed vigorously to obtain a homogenous and transparent organogel. 


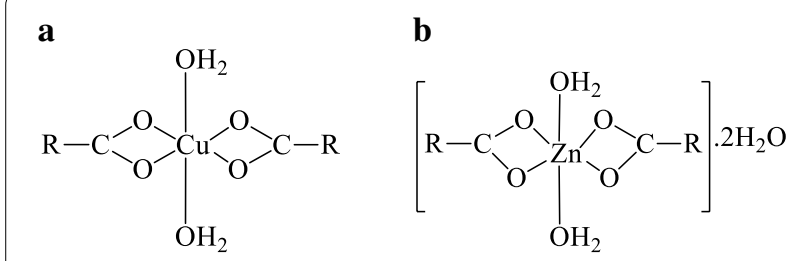

Fig. 3 Structure of: a acemetacin; b Cu(II)ACM; and Zn(II)

The organogel was allowed to de-gas overnight prior to administration.

\section{Ethics statement}

Approval of the Sydney South West Area Health Service Animal Welfare Committee was obtained. Male New Zealand White (NZW) rabbits (from the IMVS, Adelaide, South Australia) weighing approximately $2.5 \mathrm{~kg}$ were maintained on a normal chow diet throughout the studies. A non-occlusive, silastic, peri-arterial collar was positioned around the left common carotid artery [10]. The collars were left in place for $24 \mathrm{~h}$ at which time the animals were sacrificed.

The formulation was administered via laparotomy under the same general anaesthesia immediately prior to collar insertion. The rabbits $(n=4$ in each set of conditions) received either saline (positive control), IndoH (3 mg/kg), Cu(II)Indo (3 mg/kg), Cu(II)ACM (3 mg/kg), or $\mathrm{Zn}(\mathrm{II}) \mathrm{ACM}(3 \mathrm{mg} / \mathrm{kg})$. The non-collared right carotid artery was used as a control for each collared artery. The dosing for these experiments was determined on the basis of our previously published animal experiments, where there was minimal organ toxicity reported [9].

The collared segment of the left carotid artery and a $30 \mathrm{~mm}$ segment of the contra-lateral carotid artery were collected. For immunohistochemical analysis, ring sections were cut from the arteries, fixed in cold ethanol and wax-embedded, as described previously [10].

A total of 8-10 contiguous sections $(5 \mu \mathrm{m})$ and 20 random fields/section were analysed for each vessel. Mouse anti-rabbit vascular cell adhesion molecule (VCAM)-1 and intercellular adhesion molecule (ICAM)-1 antibodies (donated by Dr M. Cybulsky, University of Toronto) were used to assess endothelial expression of VCAM-1 and ICAM-1 as described previously [10].

Endothelial expression of VCAM-1 and ICAM-1 was determined by quantitative immunohistochemistry using ImagePro Plus 4.5 software (Media Cybernetics, Silver Spring, MD) [20]. The results, which represent the average positive staining above the threshold, set by a blinded experienced pathologist, for individual arterial sections are expressed as image units. Systematic sampling of each artery quadrant generated a single mean image unit value for staining for each artery. The mean of these values represents the amount of staining per treatment group, and was used for subsequent statistical comparison.

A Roche diagnostics kit (Basel, Switzerland) was used to determine plasma total cholesterol levels and HDL cholesterol levels as described [21]. The concentration of rabbit apoA-I was determined immunoturbidometrically [22]. These parameters were measured immediately prior to carotid surgery and at the time of sacrifice.

All results are expressed as mean \pm SEM. Data obtained from digital analysis were regarded as continuous variables and satisfied the Kolmogorov-Smirnov test to determine normal distribution. Statistical comparisons were then made by unpaired two-tailed Student's $t$ tests using Graph Pad Prism Version 4.0 (San Diego, CA). A value of $p<0.05$ was considered statistically significant.

\section{Abbreviations}

NSAIDs: non-steroidal anti-inflammatory drugs; VCAM-1: vascular cell adhesion molecule-1; ICAM-1: intercellular adhesion molecule; COX-2: cyclooxygenase-2; ACMH: acemetacin; IndoH: a glycolic ester of indomethacin; $\mathrm{Cu}$ : copper; Zn: zinc; SOD: superoxide dismutase.

\section{Authors' contributions}

RP, SB performed experiment, wrote the paper; AMB, RK, JEW, LC, TWH and PAL generated (Cu(II)Indo), (Cu(II)ACM), and (Zn(II)ACM); PJB, KR designed the experiment. All authors prepared the figures and manuscript. AMB and JEW looked at GI toxicity. JEW also did the formulations for the experiments and LC, TWH and PAL were also involved in the discussion of the experimental design in terms of appropriate dosing levels choices with respect to toxicity. All authors read and approved the final manuscript.

\section{Author details}

${ }^{1}$ The Heart Research Institute, 7, Eliza St., Sydney, NSW 2042, Australia. ${ }^{2}$ The School of Chemistry, The University of Sydney, Sydney, NSW 2006, Australia. ${ }^{3}$ Casbolt \& Associates Pty Ltd, Sydney, Australia. ${ }^{4}$ Lipid Research Group, Centre for Vascular Research, The University of New South Wales, Sydney, NSW 2052, Australia. ${ }^{5}$ Discipline of Pathology, D17, The School of Medical Sciences and Bosch Institute, The University of Sydney, Sydney, NSW 2006, Australia.

${ }^{6}$ Department of Cardiology, Royal Prince Alfred Hospital, Missenden Rd, Camperdown, NSW 2050, Australia.

\section{Acknowledgements}

Funding for the research was provided by Medical Therapies Ltd (now CellMid Ltd) and the Australian Research Council (ARC) through an ARC Discovery Grant (including an ARC Professorial Fellowship to PAL) and an ARC Linkage Project grant to TWH and PAL (including an Australian Postgraduate Research Award to RK), which was also supported by NatureVet Ltd. RP is the Neil Hamilton Fairley (NHMRC/NHF of Australia) Post-doctoral Fellow, PAL is an ARC Professorial Fellow.

\section{Competing interests}

The authors declare that they have no competing interests.

Received: 20 July 2015 Accepted: 25 January 2016

Published online: 06 February 2016

\section{References}

1. Wilcox CM, Cryer B, Triadafilopoulos G. Patterns of use and public perception of over-the-counter pain relievers: focus on nonsteroidal antiinflammatory drugs. J Rheumatol. 2005;32(11):2218-24. 
2. Buttgereit F, Burmester GR, Simon LS. Gastrointestinal toxic side effects of nonsteroidal anti-inflammatory drugs and cyclooxygenase-2-specific inhibitors. Am J Med. 2001;110(Suppl 3A):13S-9S.

3. Akarca US. Gastrointestinal effects of selective and non-selective nonsteroidal anti-inflammatory drugs. Curr Pharm Des. 2005;11(14):1779-93.

4. Reiss AB, Anwar F, Chan ES, Anwar K. Disruption of cholesterol efflux by coxib medications and inflammatory processes: link to increased cardiovascular risk. J Investig Med. 2009;57(6):695-702. doi:10.231/ JIM.0b013e31819ec3c7.

5. Antman EM, Bennett JS, Daugherty A, Furberg C, Roberts H, Taubert KA, et al. Use of nonsteroidal antiinflammatory drugs: an update for clinicians: a scientific statement from the American Heart Association. Circulation. 2007;115(12):1634-42. doi:10.1161/CIRCULATIONAHA.106.181424.

6. Bhatt DL, Scheiman J, Abraham NS, Antman EM, Chan FK, Furberg CD, et al. ACCF/ACG/AHA 2008 expert consensus document on reducing the gastrointestinal risks of antiplatelet therapy and NSAID use: a report of the American College of Cardiology Foundation Task Force on Clinical Expert Consensus Documents. J Am Coll Cardiol. 2008;52(18):1502-17. doi:10.1016/j.jacc.2008.08.002.

7. Weder JE, Dillon CT, Hambley TW, Kennedy BJ, Lay PA, Biffin R, et al. Copper complexes of non-steroidal anti-inflammatory drugs: an opportunity yet to be realized. Coord Chem Rev. 2002;232(1-2):95-126.

8. Jones RW, Collins AJ, Notarianni LJ, Sedman E. The comparative pharmacokinetics of acemetacin in young subjects and elderly patients. $\mathrm{Br} \mathrm{J}$ Clin Pharmacol. 1991;31(5):543-5.

9. Lay PA, Hambley TW. Use of metal complexes of antiinflammatory agents for prophylaxis or treatment of cardiovascular inflammation. PCT Int. Appl. 2007. WO 2007110755 A1 20071004

10. Puranik R, Bao S, Nobecourt E, Nicholls SJ, Dusting GJ, Barter PJ, et al Low dose apolipoprotein A-I rescues carotid arteries from inflammation in vivo. Atherosclerosis. 2008;196(1):240-7. doi:10.1016/j. atherosclerosis.2007.05.008.

11. Moore RA, Derry S, McQuay HJ. Cyclo-oxygenase-2 selective inhibitors and nonsteroidal anti-inflammatory drugs: balancing gastrointestinal and cardiovascular risk. BMC Musculoskelet Disord. 2007;8:73. doi:10.1186/1471-2474-8-73.

12. Hangarter W, Lubke A. Therapy of rheumatic diseases with a coppersodium salicylate complex compound (permalon). Dtsch Med Wochenschr. 1952;77(27-28):870-2. doi:10.1055/s-0028-1117098.
13. Sorenson JR. Copper complexes offer a physiological approach to treatment of chronic diseases. Prog Med Chem. 1989;26:437-568.

14. Lemoine P, Viossat B, Dung NH, Tomas A, Morgant G, Greenaway FT, et al. Synthesis, crystal structures, and anti-convulsant activities of ternary [Zn(II)(3,5-diisopropylsalicylate)(2)], [Zn(II)(salicylate)(2)] and [Zn(II)(aspirinate)(2)] complexes. J Inorg Biochem. 2004;98(11):1734-49. doi:10.1016/j. jinorgbio.2004.07.010.

15. Chavez-Pina AE, Vong L, McKnight W, Dicay M, Zanardo RC, Ortiz MI, et al. Lack of effects of acemetacin on signalling pathways for leukocyte adherence may explain its gastrointestinal safety. Br J Pharmacol. 2008;155(6):857-64. doi:10.1038/bjp.2008.316.

16. Haim M, Tanne D, Boyko V, Reshef T, Goldbourt U, Leor J, et al. Soluble intercellular adhesion molecule-1 and long-term risk of acute coronary events in patients with chronic coronary heart disease. Data from the Bezafibrate Infarction Prevention (BIP) Study. J Am Coll Cardiol. 2002;39(7):1133-8.

17. Dillon CT, Hambley TW, Kennedy BJ, Lay PA, Zhou Q, Davies NM, et al. Gastrointestinal toxicity, antiinflammatory activity, and superoxide dismutase activity of copper and zinc complexes of the antiinflammatory drug indomethacin. Chem Res Toxicol. 2003;16(1):28-37. doi:10.1021/ tx0200780.

18. Tabbi G, Driessen WL, Reedijk J, Bonomo RP, Veldman N, Spek AL. High superoxide dismutase activity of a novel, intramolecularly imidazolatobridged asymmetric dicopper(II) species. design, synthesis, structure, and magnetism of copper(II) complexes with a mixed pyrazole-imidazole donor set. Inorg Chem. 1997;36(6):1168-75.

19. Lay P, Hambley T, Kennedy B, Morgan Y, Kaur R, Davies N. Preparation of transition metal acemetacin complexes with antiinflammatory activity. PCT Int. Appl. 2006. WO 2006099677 A1 20060928.

20. Fang BA, Dai A, Duflou J, Zhang X, Puranik R, Bao S. Age-related inflammatory mediators in coronary artery disease (II). Int J Cardiol. 2013;168(5):4839-41. doi:10.1016/j.jicard.2013.07.157.

21. Allen JK, Hensley WJ, Nicholls AV, Whitfield JB. An enzymic and centrifugal method for estimating high-density lipoprotein cholesterol. Clin Chem. 1979;25(2):325-7.

22. Eugui J, Logrono MJ, Ruiz R, Zugaza C, Mirabel JL, Martinez C. Immunoturbidimetry of serum apolipoproteins $A-I$ and B on the Cobas Bio centrifugal analyzer: method validation and reference values. Clin Biochem. 1994;27(4):310-5.

\section{Submit your next manuscript to BioMed Central and we will help you at every step:}

- We accept pre-submission inquiries

- Our selector tool helps you to find the most relevant journal

- We provide round the clock customer support

- Convenient online submission

- Thorough peer review

- Inclusion in PubMed and all major indexing services

- Maximum visibility for your research

Submit your manuscript at www.biomedcentral.com/submit
() Biomed Central 